\title{
Mapping Accessible Paths in the City Using Collective Intelligence
}

\author{
Valmir Luiz Marques \\ Federal University of Technology - Parana \\ (UTFPR), Curitiba, Paraná, Brazil \\ valmirluizmarques@gmail.com
}

\author{
Alexandre Reis Graeml \\ Federal University of Technology - Parana \\ (UTFPR), Curitiba, Paraná, Brazil \\ graeml@utfpr.edu.br
}

\begin{abstract}
New information and communication technologies (ICTs) have an increasingly stronger role in people's lives, especially after the commoditization of smartphones. They affect many aspects of everyday life, including urban mobility. Some applications, including Waze, benefit from the collective intelligence (CI) of the crowds to gather the information they need to provide users with good advice on the routes to follow. But they are mainly focused on roads and streets, giving little information on the quality of sidewalks, which are essential to pedestrians, people on wheelchairs and blind people. With the intention to improve the mobility of citizens with special needs, we developed the prototype of an application that allows users themselves to update accessibility maps, tagging obstacles and also indicating the existence of resources that contribute to improve the mobility of people with special needs in urban spaces. Tests in a controlled environment helped to debug the application's functionalities, before members of the intended target group of users were finally exposed to it. Results are promising, as users were able to include relevant data by themselves and seem motivated to keep doing so, due a sense of utility, social facilitation or simply due to altruism, as anticipated by the CI literature. One unexpected outcome was that impaired users are more excited about the potential the application has to give visibility to the challenges they face than with the actual improvement it can bring to their mobility.
\end{abstract}

\section{Introduction}

People with disabilities are discriminated and prevented from fully exercising their citizenship, in many ways. One of them is by not being provided with adequate means to safely move around in the city [1], [2]. Sidewalks without adequate accessibility hinder the mobility of wheelchair users and visually impaired people, among others, even on short trips, such as going from one street corner to the next, on a sidewalk.

New information and communication technologies (ICTs) can contribute to coordinate people's joint work towards their goals, reducing cultural and social barriers while maximizing the possible outcomes of the collaboration of many individuals [3]. As Nagar [4] points out, collective intelligence $(\mathrm{CI})$ systems are increasingly used to get ideas out, develop projects, make forecasts, and get things done, based on efforts developed by a collective of people submitted to challenges that bring them together to work on a project.

When it comes to upkeeping collaborative mapping systems, the collective intelligence of users is very effective. Users can perform the marking of occurrences or provide feedback, which is very useful for map updating [5]. This already occurs, for example, in Waze (a social network with GPS system) and OpenStreetMap among other navigation systems [6].

A widely used term in the literature, to refer to gathering and updating information from users through mobile devices is crowdsensing. It was used for the first time in 2007, as noted by Ganti et al. [7]. This is a technique by means of which a group of individuals collectively shares data and extracts information to measure, map, analyze or estimate any information that may be of common interest [8].

In preparation for the development of the application reported in this paper we carried out two systematic literature reviews (SLR) to investigated the state of the art of "accessible maps" [9] and the "incentives/ motivations" for users to contribute with their updating (this second SLR paper is currently going through a journal's editorial process and is still not publicly available). The purpose of carrying out such reviews was to understand how far others had already gone with projects with similar intents, in order to try and benefit from their experience in giving one further step towards improving urban mobility for people with disabilities, specially those with any sort of motor or visual impairment. Our SLRs have shown that, although a lot has already been done in the sense of building accessibility maps that can support people with disabilities, and that even collective intelligence has been used for that 
in several occasions, previous applications do not update their maps dynamically, which reduces their attractiveness to users interested in using them to move around and also to those who wish to help updating them, because they are not able to see the result of their actions, straight away. Another thing that has not been attempted yet is to support users with different special needs, all at once. Previous works report on applications focused on wheelchair users only, or blind people only. They never deal with these different groups conjunctly. We understand that this may cause an increase in the complexity of the system, but, at the same time, an application that is concerned with diversity provides for a more inclusive environment, contributing to people's better understanding of each others' problems and needs. That way, the application may also become an important awareness tool to make society aware of the challenges some of its citizens face to move around in their daily routines. This is, in fact, the main reason for us to also try and involve people with no disabilities in the collective effort to keep the application's maps updated.

To accomplish that, the application will collect, organize and use location-based information, obtained by means of crowdsensing and collective intelligence efforts, to populate accessibility maps concerning sidewalks and other public spaces.

The remaining sections of this paper are organized as follows. A brief literature review, discussing related work, on collective intelligence, mobile crowdsensing and types of incentives for collective intelligence is presented next. Then, some important information on the developed application is provided, and finally the results obtained from tests with a controlled group and with intended target users are discussed.

\section{Related work}

Related work is discussed in this section, which helped assessing features that needed to be included in the application and issues that needed to be considered, in order to involve people, motivating them to collaborate, feeding the system with the required information for it to work accordingly.

\subsection{Collective Intelligence (CI)}

Lévy [10] defines CI as a form of intelligence that emerges from the collaboration and competition of many individuals. Malone et al. [11] consider it to be something that happens in a group of individuals acting collectively in a way that seems intelligent. CI represents some sort of shared intelligence resulting from the collaboration of many individuals [11]. It has been around for a long time [10]. However, currently, the types of CI that evolve more rapidly are those that benefit from the Internet as a platform for the coordination and performance of activities involving collectives of people [12].

The individual decisions made by participants in communities where CI takes place are, many times, aggregated in an attempt to produce a high-quality collective decision, comparable to the judgment of experts [13].

According to this, CI may provide a useful way to help to update collaborative map applications, where the information generated by users can be used to generate accessible routes or to provide information about accessible venues to people with disabilities.

\subsection{Mobile Crowdsensing (MCS)}

With the omnipresence of mobile devices, in a mostly connected world, mobile phones have become a powerful tool to collect information and make it available to participatory systems, using sensor data and the Internet, on a social scale [7].

According to Laurindo and Feitosa [14], MCS consists of a new sensing paradigm, leveraged by the power of mobile devices, taking advantage of devices that "follow" users wherever they go to acquire important local knowledge and share this knowledge within a social scale [7]. Silva et al. [15] argue that MCS systems allow people to share useful data about the context, at any time, becoming potential detection sources on a global scale.

Depending of the type of application, and the resources of the used device, MCS can be classified as "participatory" or "opportunistic" [7]. It is participatory when the users know and understand the context of the application and are personally involved, or at least authorize access to information their devices generate, i.e., data is provided voluntarily. The opportunistic approach takes advantage of other online social interactions, for example, check-ins performed in a social network, such as Foursquare, Instagram or Twitter [15]. In this case, the user is not necessarily aware of the real context of the application that will use the generated data. S/he may not even be aware that his/her data is being used for any other purpose than his/her direct intention, at all.

In this project, both types of MCS are used. Participatory crowdsensing helps to collect accessibility data provided directly by users to the application, and opportunist crowdsensing, is used to harness accessibility data from the wheelmap.org platform, complementing data that is voluntarily and directly provided by users. MCS also helped users to take photos of specific markers, tagging them to the user's location, based on the device's GPS functionality. That means up to date information becomes available and accessible straight away. 


\subsection{Motivation for Collective Intelligence}

To keep users engaged in contributing to a platform, mainly when participatory sensing is used, it is essential that they see, in the application, a good reason to use it, either for their own benefit or the benefit of others they care for.

However, even in cases where opportunistic sensing is used, it may be necessary to establish mechanisms to stimulate specific actions by users. Although, in general, these actions are not perceived by the users as being associated with the purposes intended by the sponsor of the sensing [16].

Although the literature shows some concern about the possibility of a crowdsourcing venture being sabotaged by individuals with conflicting intentions [17], we have, so far, focused only on providing people with good reasons to contribute to the collective effort. Malone et al. [11] refer to the love, glory and money genes that could be used to try and align crowdsourcees' interests to the crowdsourcer's objectives. Considering that we have no means of paying people for their support in keeping map information up-to-date, we included features in the application that try to motivate them to collaborate for love and/or glory, primarily. We believe that, by means of the included features, and others that we still plan to include in the future, we foster the individuals' cooperative behavior and, at least, do not provide them with any specific incentive for non-cooperative or malicious use of the application. However, the resilience of the developed approach to non-cooperative behavior still needs to be assessed and will possibly require further attention in future versions of the application.

In Table 1, below, we highlight some incentive and motivation strategies, aimed at keeping users active in the application and contributing the needed data for the application's operation. These incentives were compiled as a result of one of the systematic literature reviews (SLR) mentioned in the Introduction section.

Each of these concepts was considered in the development of the application. They were used whenever we found suitable to try to increase the motivation to participate. The way this was done will be further explored in section 4 , ahead.

In the following section, we discuss the adopted methodological procedures.

\section{Methodological procedures}

In this section, we explain the research steps: the definition of the features and requirements for the application, the identification of suitable motivation strategies to stimulate participation in the crowdsensing effort, the development of the application, its initial debugging, in a controlled environment, and its presentation to target users. Figure 1 shows all these steps.

Table 1. Motivation strategies generally used in crowdsensing systems

\section{Gamification}

Defined as the game-design-based incentive process, which intends to recruit users to solve problems, by including game elements in the tasks [18].

\section{Social incentive (friends)}

Involves encouraging one's friends to also contribute, carrying out sensing tasks, taking advantage of the social bonds among participants to increase the number of collaborators [19].

\section{Ranking / hall of fame}

Users with the highest scores are put in the spot light [20], exploring the glory gene of CI motivation, as proposed by Malone et al. [21].

\section{Altruism}

Love for one's neighbors, or disinterested concern, philanthropy or community sense [22]. Explores the love gene, as identified by Malone et al. [21].

\section{Storytelling}

Users are led to tell stories or make reviews, sharing them with other users [23].

\section{Sense of utility / value added}

Users are led to understand that the system can bring benefits to themselves and to other people. This may increase the likelihood of participation in performing tasks, compared to systems perceived by users as mere "objects of interest" [24].

Situation at the place of occurrence

Possibility of visualizing a situation that happened exactly at a specific spot can stimulate users to contribute with additional annotation [25].

\section{Social factors}

Takes into account socioeconomic issues, commercial interest or public good [26].

\section{Social transparency}

Use of real names and identities in crowdsensing applications [27].

Source: authors' previous work (still not published)

\subsection{Theoretical phase}

The preliminary characterization of the features was based on the SLR about "accessible maps", in which the state of the art of this type of system was identified, as well at the limitations or gaps in such systems, which we tried to eliminate by including some additional features and making the application more inclusive of different kinds of disabilities.

The second SLR, about "motivation and incentive methods in mobile crowdsensing systems", provided us 


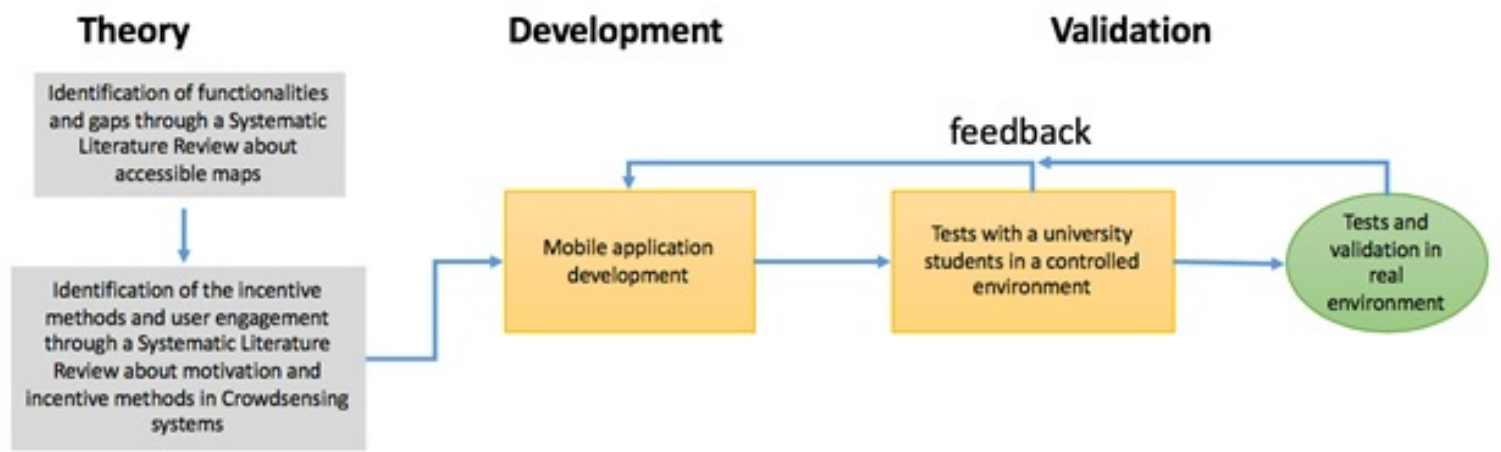

Figure 1. Steps for the development and validation of mobile application

with important hints on how to motivate users, as already discussed and shown in Table 1.

\subsection{Development phase}

The mobile application, whose features were based on the results obtained in the literature review phase, was developed for Android platform. This operating system was prioritized because, according to an IDC report [28], it is used by $85 \%$ of smartphone or tablet users. The operating system also has the "talkback" accessibility feature, which provides users with audio feedback from any component in the application, such as labels, text boxes or images (as long as it is previously programmed for that). This is an important feature, particularly useful in the case of visually impaired users. The application was developed in compliance with the WCAG (Web Content Accessibility Guidelines) accessibility standard. Text size, color contrast and audio feedback for any text were all taken into consideration. Figure 2 shows an example of configuration of the description of an image, so that it can be handled by the operating system's talkback feature. When browsing the screen and clicking on the image, the user listens to the audio related to the text, as configured in the "contentDescription" attribute of the programming language.

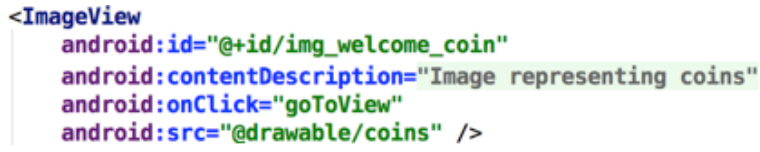

\section{Figure 2. Example of textual description of an image}

\subsection{Test phase}

The controlled environment testing stage was carried out with a group of Information Systems undergraduate students from the Federal University of Technology Parana, in the South of Brazil. Eight groups of three students were formed and each group was responsible for covering one complete city block, around the university, adding accessibility information to the map. In total, eight city blocks were mapped, which was performed in less than half an hour.

A questionnaire was then sent to each of the students, asking them about their perception on the usability of the mobile application and for suggestions of new features and improvements to the existing ones.

The results of the controlled environment test are presented in section 5. After this test, the application was improved, and its detected bugs were fixed. Then the application was exposed to target individuals (wheel chair users) for preliminary field assessment, which is reported in section 6 .

\section{The mobile application}

The prototype of the mobile application developed for this research project is described in this section, with respect to its technical characteristics, architecture and main features.

\subsection{System architecture}

The mobile collaborative map application was developed based on the Android operating system.

For the application to work, the GPS (Global Positioning System) function needs to be activated, as it is responsible for presenting the map relative to the users' location. Figure 3 shows the basic scheme of the application's operation. Through participatory MCS, users of the application mark accessibility points (existence of curb ramps, audio traffic signals, tactile strips on sidewalks, bus stops etc.), inaccessibility points (pavement holes, construction, stairways etc.), in addition to the type of existing sidewalk pavement and the perceived ease of locomotion on it.

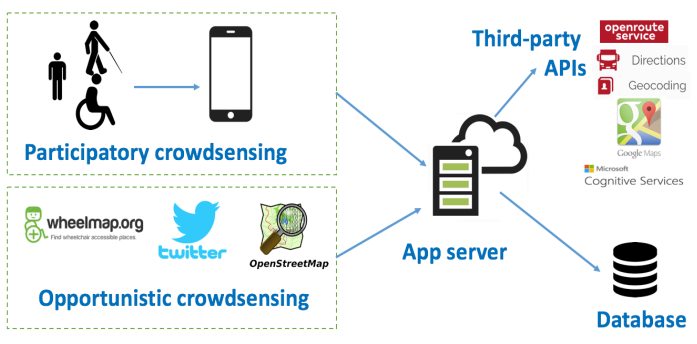

Figure 3. Application's architecture model 
Opportunistic MCS is also used to extract data from social networks and other collaborative map applications such as Wheelmap.org. This data, along with third party APIs, are used to comprise the application's features. Third-party resources helped reducing development time.

The next section introduces some of the main features of the application.

\subsection{Main Features of the Application}

User settings: the application has a section for the user's registration and provision of basic personal data for the user's profile. The registration is mandatory, because social transparency (use of real names and the identification of users) is required, in order to make contributions more credible and reliable [27]. Figure 4 shows the application screens for the sign-up process.

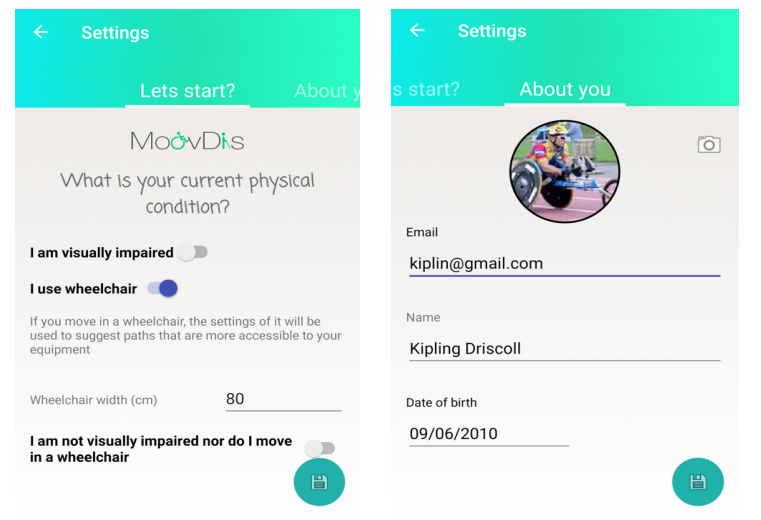

Figure 4. User sign up

Visually impaired users can use Android's talkback accessibility feature to read the screen or expand the displayed text of labels and other screen components.

In the settings screen, the user can explicitly tell if $\mathrm{s} / \mathrm{he}$ is a wheelchair user, if s/he is visually impaired or has no moving limitations. Settings are used to adjust application features according to user characteristics. For example, visually impaired users can interact with a virtual assistant, using natural language.

Map and accessible routes: after the sign-up process, the user can access and use the application. When accessing the map, the user's current location area is displayed, as well as occurrences marked in the neighborhood, as shown in Figure 5.

It is possible to search for an address or place, to trace a pedestrian route, to mark positive occurrences (e.g. sidewalk with curb ramps, place with accessible restroom, tactile strips on the sidewalk, parking lot with spaces reserved for disabled people, traffic light with audio signaling etc.), to mark negative occurrences (e.g. barrier, construction in progress on the sidewalk, semi-permanent obstacle on the sidewalk, obstructed walkway, elevation, narrow sidewalk, danger to the blind etc.), as shown in Figure 6. It is also possible to mark the type of sidewalk and the walkability difficulty level as perceived by the user, as shown in Figure 7. This sidewalk type marking feature was developed after the initial controlled environment tests, as a suggestion from one of the participants, who noted that it could be helpful to identify the pavement type in advance and, consequently, allow a better planning of the journey, considering that certain types of pavement have a less rugged surface than others.
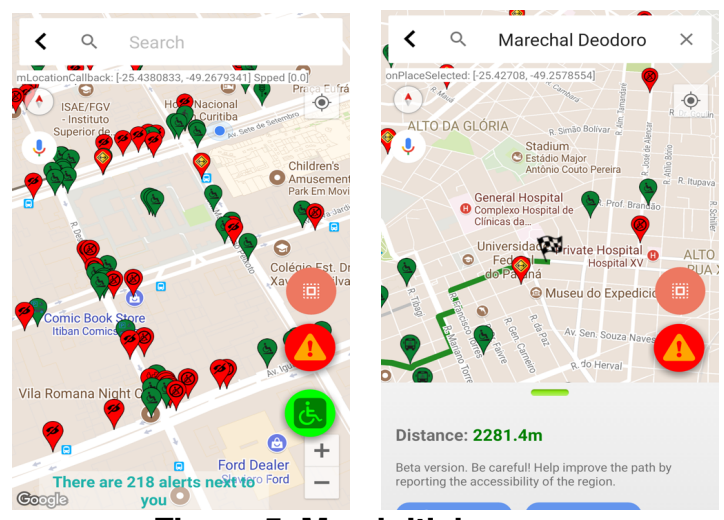

Figure 5. Map initial screen and route generation

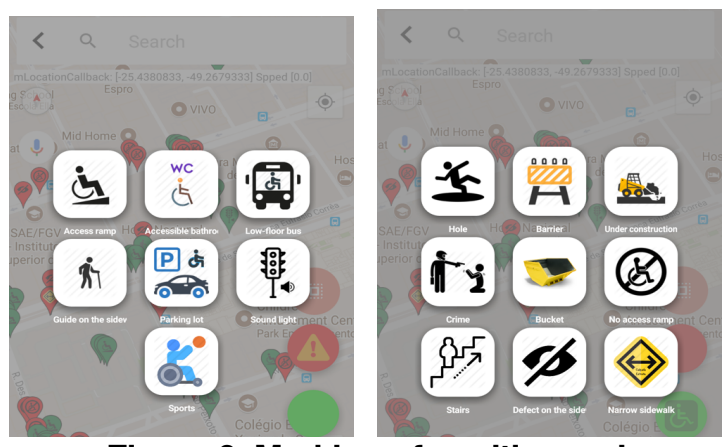

Figure 6. Marking of positive and negative occurrences

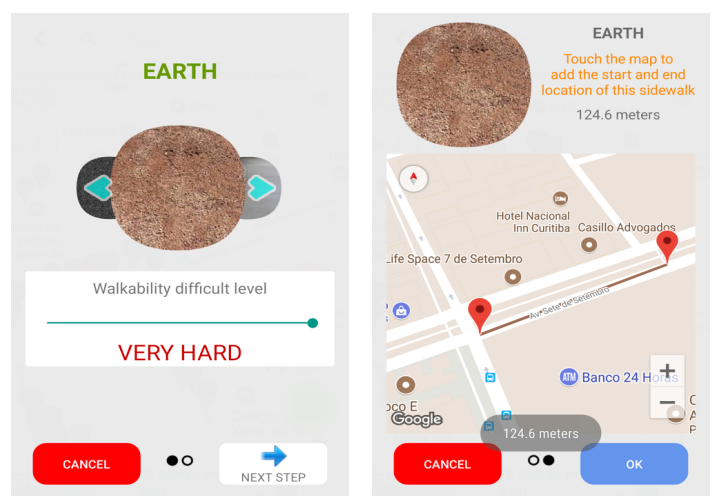

Figure 7. Marking of sidewalk type 
In this step, mechanisms of incentive were used, including highlighting a situation at the place of occurrence [25] and altruism [22], to stimulate the user to mark occurrences at the time and place where they occur, helping other users with such information.

Scoreboard: with the aim of stimulating competition and encouraging users to contribute more to the platform, gamification [18] and ranking/hall of fame [20] incentive mechanisms were used to show the individual score and the overall score of users who contributed to the platform. For each contribution that the user makes, s/he accumulates virtual coins.

In the overall scoreboard, the user's position is shown relative to others. Top "citizen" contributors earn more coins and are ranked higher. Here, we explore the competition among collaborators. However, this scoreboard is renewed every week, so there is a chance that new users will appear at the top of the ranking and not only those who have been contributing for a long time.

The individual scoreboard, on the other hand, stimulates users to compete against themselves. As observed by Bergendahl et al. [29], although group competition encourages collaboration, individual competition is an important factor of intrinsic motivation. Not all people are motivated by the competition against others. There are those who prefer to engage in personal challenges. Therefore, it is also important that there are "prizes" for one's individual achievements. In this scoreboard, the progress of the user is shown over time. Users are classified according to levels: beginner, focused or advanced. The intention is that users wish to appear as advanced users, as time goes by. The history of user contributions is also shown. Figure 8 shows the overall and individual scoreboard screens.

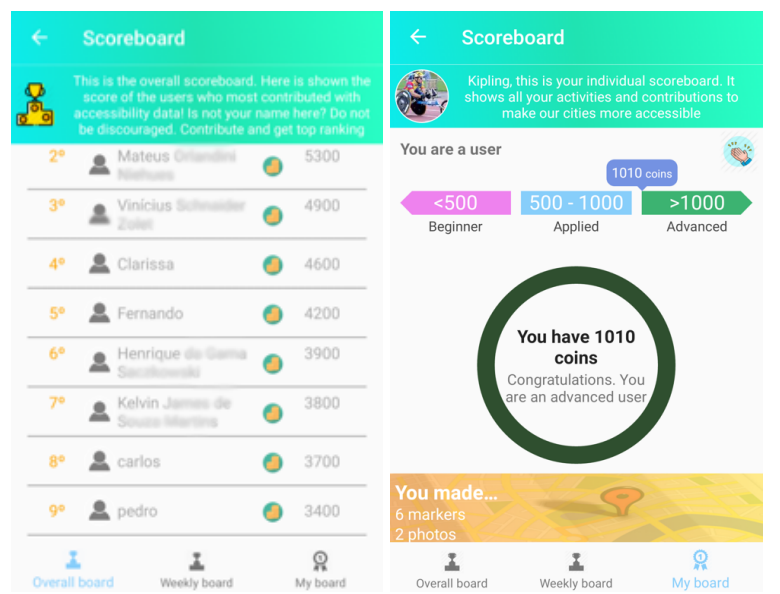

Figure 8. Incentive mechanism based on rankings with scoreboards

Feeds: by means of this feature, we feed users with posts and news published in social networks, with subjects related to urban mobility, accessibility and inclusion. Here, again, we try to explore social transparency [27], the sense of utility [24] and storytelling [23], where social networking data can help users become more informed about urban mobility.

Virtual assistant (VA): with the aim of facilitating users' interaction with the application, improving his/her experience, especially in the case of visually impaired users, a virtual assistant was developed. The user can use natural language to interact with the application. The main features and commands are:

"Where am I?": the virtual assistant tells the user's current location;

"There is a barrier here!": the virtual assistant marks an occurrence in the map;

"I want to go to ...": the virtual assistant traces an accessible route from the current location to the desired destination and orally guides the user along the way;

"What are the occurrences in the region?": the virtual assistant tells about markers in the neighborhood.

Other features: other ancillary features were developed, in order to stimulate the social interaction of users, exploring social incentives [19]. For example, the application allows users to invite friends to use the platform and share the routes they take with other users, allowing for interaction and feedback. Another feature that explores social factors [26] is the push notification. Users receive a daily push notification with content that indicates the accessibility needs and asks the user to include accessibility markers related to his/her current location.

\section{Test in a controlled-environment}

The fact that we evaluated and tested the system in a controlled environment, with undergraduate students who are not physically or visually impaired, allowed us to gather some initial results, which were very important for the evolution of the application. There was some debugging and testing of the application that we thought we could spare our target groups of users from. We believed that they should have access to an application that was as functional and efficient as possible in providing them with the expected results and did not want to risk losing their enthusiasm due to any issue that we could fix ahead of involving them. Non-end-user involvement, in this phase, also helped protecting a more fragile group of users, in a stage we still had many things to fix. We were aware that there would be issues that could possibly only be brought up by the targeted main end-users (citizens with special mobility needs), but we thought that non-end-users could help us improve the application prior to that.

So, a class of undergraduate students was divided into 
groups in order to map accessibility around the university campus. Upon returning from the field, a feedback questionnaire was applied to the eight groups of students who tested the application and gave their impressions about it. Based on their feedback, we were able to observe that: the application was easy to install and moderately successful in its purposed use, the interface was user friendly, and users were willing to share it with friends, as shown in Table 2.

Table 2. Application evaluation

\begin{tabular}{l|c}
\hline \hline \multicolumn{2}{c}{ How easy was the installation of the app? } \\
\hline \hline Extremely easy & $33,3 \%$ \\
\hline Very easy & $33,3 \%$ \\
\hline Moderately Easy & $33,3 \%$ \\
\hline Difficult / Very Difficult & $0 \%$ \\
\hline \hline
\end{tabular}

How friendly is the application interface?

\begin{tabular}{l|c}
\hline \hline Extremely friendly & $0 \%$ \\
\hline Very friendly & $100 \%$ \\
\hline \multicolumn{2}{c}{ Moderately friendly / Unfriendly } \\
in performing its functions? \\
\hline \multicolumn{2}{c}{$0 \%$} \\
\hline Extremely successful & $0 \%$ \\
\hline Very successful & $33,3 \%$ \\
\hline Moderately successful & $66,7 \%$ \\
\hline Unsuccessful / Nothing successful & $0 \%$ \\
\hline \hline \multicolumn{2}{c}{ What is the probability of recommending the } \\
application to your friends and colleagues? \\
\hline \hline Very likely & $66,7 \%$ \\
\hline Moderately likely & $33,3 \%$ \\
\hline Not likely & $0 \%$ \\
\hline
\end{tabular}

The test phase in a controlled environment was important to identify how the application behaved in different operating system versions and using different smartphone models, from different manufacturers. Photo capturing, for example, was not working in some devices and the test helped to detect and solve the problem.

As expected, and according to Figure 1, the test and validation step generated new ideas and improvement opportunities that required further development, starting a new cycle of continuous evolution.

The possibility of dragging a marker to the correct location position on the map and marking the type of pavement of sidewalks were both functionalities that were added in response to the participants' comments (see Table 3 , for some of the questions and answers from participants in the test).

After the test, with bugs having been fixed and new features added, the application was presented to users belonging to the main target group, in a real environment.
Table 3. Feedback from users

Q: Has the application ever crashed or presented any abnormal behavior during use? If yes, in what feature?

A1: I had trouble updating the markers on the street as I did not know if there was already any tag for a specific place.

A2: I had trouble adding photos to the markers. The application takes photos, using the device's camera, the photos are saved on my phone, but it was not clear if they were being added to the tags.

Q: When you marked occurrences on the street, were there any situations that you could not mark because they had not been planned in the app?

A1: The lack of an icon for tactile sidewalk strips for the visually impaired and an icon for poor curb ramps (that make it difficult for wheelchair users to progress, without a helper to push).

$\mathrm{Q}$ : What are your recommendations for improving the application?

A1: There should be an option to drag a marker and drop it at the precise location where it should be, because often the GPS is not that accurate and ends up erroneously marking the place.

A2: Can't mark the type of paving on the sidewalk. And I believe the type of paving makes a huge difference for someone in a wheelchair.

A3: Allow the inclusion of temporary markers, which would need to be checked after a certain time or could just vanish.

\section{First perceptions of wheelchair users}

After the tests in the controlled environment, we were able to perform qualitative tests and obtain user perceptions in the use of the application in a real environment. A group of wheelchair members of the Association of Physically Disabled People of Paraná (ADFP), in Brazil, agreed to be introduced to the application and provide feedback.

We visited the association three times before we gained the wheelchair users' trust and were able to collect the data we needed. In our first visit, we presented the application to the president of the association and one of the managers. Functionalities were demonstrated, focusing on the ones that allow for collaboration and stimulate collective intelligence efforts to populate the database. After this presentation, the two members of the association (who are also physically disabled) were asked if they felt that the developed artifact made sense and would be useful to the members of their association. They acknowledged interest and a second visit was scheduled to present the application to some potential end users. In this presentation, the application was demonstrated to five users (4 wheelchair 
and a crutch users). They all agreed to test the application in the field. They used the application for a week, generating accessibility data based on their mobility over that period. Then, an evaluation questionnaire was applied, generating the results that are presented next.

\subsection{Perceptions about functionality and interface}

In order to assess the perceived ease of use of the interface, and also the usefulness of the developed features, some questions were asked to the participants.

Users were asked how friendly the application interface was. Two of them considered it extremely friendly and one claimed it was very friendly. The last two said it was moderately friendly. Although there is clear opportunity for improvement, the interface seemed sufficiently functional for the users.

Users could rate each of the functionalities, based on their perception of usefulness. The possibilities were: 1 (little / not useful), 2 (useful) or 3 (very useful). It was also possible to mark the option $\mathrm{n} / \mathrm{u}$ (did not use). Table 4 shows the consolidation of user evaluations. For this analysis we considered a feature was useful when it scored at least 2 on average, disregarding the "not used" answers some users gave to some functionalities.

Table 4. Evaluation of the application's functionalities, according to users' perceptions

\begin{tabular}{|c|c|c|c|c|c|}
\hline Feature & 1 & 2 & 3 & $\mathbf{N} / \mathbf{U}$ & Rate \\
\hline Maps & - & - & $100 \%$ & - & 3,0 \\
\hline Trace a route & - & - & $60 \%$ & $40 \%$ & 3,0 \\
\hline $\begin{array}{l}\text { Mark and view } \\
\text { occurrences on } \\
\text { the map }\end{array}$ & - & $20 \%$ & $80 \%$ & - & 2,8 \\
\hline $\begin{array}{l}\text { Mark and view } \\
\text { the type of } \\
\text { sidewalk }\end{array}$ & - & $20 \%$ & $80 \%$ & - & 2,8 \\
\hline $\begin{array}{l}\text { Accessibility of } \\
\text { venues }\end{array}$ & - & - & $100 \%$ & - & 3,0 \\
\hline Feeds & - & $20 \%$ & $40 \%$ & $40 \%$ & 2,66 \\
\hline Events agenda & $20 \%$ & $40 \%$ & $40 \%$ & - & 2,2 \\
\hline Virtual assistant & - & $40 \%$ & $40 \%$ & $20 \%$ & 2,5 \\
\hline Scoreboard & - & $20 \%$ & $80 \%$ & - & 2,8 \\
\hline $\begin{array}{l}\text { My routes - view } \\
\text { and share }\end{array}$ & - & $20 \%$ & $60 \%$ & $20 \%$ & 2,75 \\
\hline Invite friends & $20 \%$ & $40 \%$ & $40 \%$ & - & 2,2 \\
\hline
\end{tabular}

Based on the answers, all functionalities were considered useful. It is important to highlight that some of them have a functional purpose, while others exist to stimulate usage and increase the number of users of the application.

The functionalities that obtained the highest scores were functionalities that bring the accessibility information to the users (maps, marking occurrences, marking the type of the sidewalk, accessibility of venues and scores) and that foster "collective intelligence". The features with lower scores were ancillary features, aimed at attracting more audience to the application and encouraging users to contribute more (feeds, event calendar, invite friends to the application). It is reasonable that users consider these ancillary features less important. After all, they are not useful to get what they need from the application, directly. They are there to stimulate consistent use and to help to improve the quality and quantity of data in the application, over time.

Some functionalities were not evaluated by the users, simply because they were not used by them. One possible reason for the "trace a route" functionality not having been used that much, although it is planned to be a central functionality, is that the application still does not have much accessibility data included, which can make the route result unreliable.

\subsection{Motivation to use the application}

In an attempt to evaluate the effectiveness of the motivation mechanisms employed in the application, and discussed in the previous sections, respondents were asked about what motivated them to use the application and what would motivate them to continue using it in the future. They had a free text input field to answer that. A qualitative analysis was performed of the answers, trying to classify them according to the motivations presented in Table 1. Three motivators stood out: altruism [22]; sense of utility and added value [24]; and social factors [26]. Altruism is important here, because accessibility is an issue that should always concern our society, after all, everyone has the right to it. The sense of utility and added value appears because participants understand that the data they generate is useful for the whole community. And, finally, social facilitation was a perceived motivator because the problems of the city concern the whole society and, especially, public agencies.

Finally, to check if they were also stimulated by social incentives [19], users were asked about how likely they would refer the application to other people. Four of the five respondents said that it was extremely or very likely that they would invite their friends to use the application, at some point.

One think that should be highlighted is that the participants all reported that it would be useful to include users without any disabilities as intended users of the application. That was something we had already planned for, because it would mean more people contributing to populate the database of accessible information, but mainly because that would make more people aware of the 
difficulties wheel chair users and other impaired citizens have to move around the city. In fact, that was perceived as the main added value [24].

\subsection{Collective intelligence in action}

Collective intelligence principles were used, initially, to aggregate the markings performed by different individual users of the application to populate accessibility maps. The collective effort serves as a basis for the generation of useful and up-to-date information for all users, providing a superior solution, compared to the static maps proposed in some of the literature. A new marking on a map represents a valuable input to avoid a bad path to be suggested to a user or to allow a user to know in advance, how accessible an establishment $\mathrm{s} / \mathrm{he}$ wants to visit is.

The generation of new information on maps, besides supporting other users, also contributes to the identification of city problems. If used by public agencies, it can help to solve problems for all citizens. Once again, collective concerns are prioritized, as collective intelligence helps local governments to decide on interventions in the city that will make them a better place for all citizens, paying attention to the needs of weak groups that usually do not have their needs properly expressed and, therefore, accounted for.

\section{Conclusion}

The prototype of an accessibility and urban mobility application was presented, which was developed using concepts of CI with the intent to facilitate the locomotion of physically or visually impaired citizens in the city.

It is plausible to say that the prototype of the application reached its proposed goal, first in the generation of CI, providing important inputs to achieve a greater objective that is social inclusion and impact in the lives of people with physical disabilities, allowing them to plan their moving around the city more efficiently.

Initial testing in a controlled environment allowed us to improve some features and create others to insure the usability of the application.

The involvement of users was essential to the validation of the solution. Although one cannot measure how much impact the application will have on people's lives, at this stage, there has been a clear interest in the outcome of this research, especially noticeable after the presentation of the application to members of the association of wheelchair users (ADFP). There is a great deal of motivation and expectation that the application will help them with their mobility issues, on a day-to-day basis, but especially that it will help to make the city's mobility problems more visible, from the perspective of disabled people. They believe it could help them to make their "voice" heard. This was an interesting and unexpected result: we intended to improve impaired people's mobility. They are happy with that, but more than anything else, they feel that the application will allow them to make themselves noticed by other people and the authorities. This is an unplanned externality that may prove more important than all the intentionally included functionality.

So, although we could claim that the contribution of this paper is the presentation of an artifact that was built taking into account the issues and gaps that appeared in the literature review about accessible maps, crowdsensing and collective intelligence, if the application is embraced by a community, that may be perceived as a smaller contribution, when compared to giving "voice" to the unheard, making other people aware of the challenges they face and including them, a little more, into our society.

The two rounds of tests, after the SLRs, brought the wisdom of three different groups into play: the authors in the field who concerned themselves with the issue over time and wrote papers about it, Information Systems undergraduate students with some knowledge about how systems should perform, and people with special needs, showing what is really relevant to them. Three layers of CI piled one on top of the other, but there is a possibility of interacting among them, in future rounds of improvement of the application.

People with visual disabilities haven't yet been heard, at least not through a formal presentation of the application, specifically to them. So, most of the concerns with respect to making the application compliant with WCAG have not been tested to exhaustion. A future work could focus on a sample of users with visual impairment, to make sure that their needs are thoroughly taken care of. Future work could also concern with people with different levels of reduced mobility and consider the equipment they have access to. Wheel chair users that have their wheelchairs equipped with electric motors will find it easier to take more inclined routes than those who depend on the strength of their arms to push their chairs around, for example.

We are still far from being able to assess the effectiveness of the application to different targeted groups. A reviewer, who had access to a draft of this paper, reminded us that an important evaluation criterion should also be: how well-planned routes match the handicap of the user. S/he also thinks that it might be worthwhile to discuss the level of handicap beyond binary choices. We totally agree with that and hope we can refine the application and get there, in the near future. For now, we are happy with the fact that people who once felt as second-class citizens believe our software has the potential to help them to become more noticeable to society. The few wheel chair users that were introduced to the application, unanimously, agreed that the application can give them more "voice". If that really happens, our effort will have already paid off, although we have to admit that empowering them had not 
occurred to us as something we could do. It turns out we were trying to solve a problem than now seems clearly much smaller than what we can actually help to do!

\section{References}

[1] V. Broadus, "Mobility for disabled people is a challenge to be overcome in Brazil," mobilize brasil, Mar-2012. [Online]. Available: https://goo.gl/WQqddZ. [Accessed: 15-Nov-2016].

[2] T. Leitão, "Accessibility is a challenge for disabled people all over the country," exame.com, Nov-2012. [Online]. Available: https://goo.gl/aVbH6P [Accessed: 18-Nov-2016]

[3] T. W. Malone and M. S. Bernstein, Eds., Handbook of collective intelligence. Cambridge, Massachusetts: MIT Press, 2015.

[4] Y.Nagar, "Designing a collective-intelligence system for evaluating complex, crowd-generated intellectual artifacts," Conference on CSCW'13, p. p.73-76, 2013.

[5] N. R. Budhathoki and C. Haythornthwaite, "Motivation for Open Collaboration: Crowd and Community Models and the Case of OpenStreetMap," American Behavioral Scientist, vol. 57, no. 5, pp. 548-575, 2013.

[6] C. Prandi, V. Nisi, P. Salomoni, and N. J. Nunes, "From gamification to pervasive game in mapping urban accessibility," presented at the CHItaly 2015 Proceedings of the 11th Biannual Conference on Italian SIGCHI Chapter Pages 126-129, 2015, pp. 126-129.

[7] R. Ganti, F. Ye, and H. Lei, "Mobile crowdsensing: current state and future challenges," IEEE Communications Magazine, vol. 49, no. 11, pp. 32-39, 2011.

[8] D. Peng, F. Wu, and G. Chen, "Pay as How Well You Do: A Quality Based Incentive Mechanism for Crowdsensing," in MobiHoc '15, Hangzhou, China, 2015, pp. 177-186.

[9] V. L. Marques and A. R. Graeml, "Accessible maps and the current role of collective intelligence," GeoJournal, Apr. 2018.

[10] P. Lévy, "A inteligência coletiva: por uma antropologia do ciberespaço " [Collective intelligence: for an anthropology of cyberspace]," in [The Collective Intelligence], 4. ed., São Paulo: Edições Loyola, 2003.

[11] T. W. Malone, T. Atlee, and P. Lévy, Collective intelligence: creating a prosperous world at peace. Oakton, Va.: Earth Intelligence Network, 2008.

[12] A. Kleiner, "Os 3 segredos improváveis das melhores equipes [The 3 unlikely secrets from the best teams]," Revista HSM, 04-Aug-2014.

[13] J. Chamberlain, U. Kruschwitz, and M. Poesio, "Motivations for Participation in Socially Networked Collective Intelligence Systems," presented at the CI 2012, Social and Information Networks; Physics and Society, Cambridge, MA, 2012, p. 8.
[14] J. J. de V. Laurido and E. L. Feitosa, Segurança em Mobile Crowd Sensing [Mobile Crowd Sensing Security], 1st ed., [Book of minicourses presented in the XVth Brazilian Simposium of Information Security and Computational Systems]: SBC, 2015.

[15] T. Silva, P. S. Vaz De Melo, J. Almeida, and A. F. Loureiro, "Large-scale study of city dynamics and urban social behavior using participatory sensing," IEEE Wireless Communications, vol. 21, no. 1, pp.42-51, Feb. 2014.

[16] Y. Zhan, Y. Xia, Y. Liu, F. Li, and Y. Wang, "IncentiveAware Time-Sensitive Data Collection in Mobile Opportunistic Crowdsensing," IEEE Transactions on Vehicular Technology, vol. 66, no. 9, pp. 7849-7861, Sep. 2017.

[17] M. Harris, "How A Lone Hacker Shredded the Myth of Crowdsourcing," Medium, 2015. [Online]. Available: https://goo.gl/1R6h6D. [Accessed: 01-Jul-2018].

[18] J. Brito, V. Vieira, and A. Duran, "Towards a Framework for Gamification Design on Crowdsourcing Systems: The G.A.M.E. Approach,” 2015.

[19] G. Yang, S. He, Z. Shi, and J. Chen, "Promoting Cooperation by the Social Incentive Mechanism in Mobile Crowdsensing," IEEE Communications Magazine, vol. 55, no. 3, pp. 86-92, Mar. 2017.

[20] W. Rafelsberger and A. Scharl, "Games with a purpose for social networking platforms," 2009, p. 193.

[21] T. W. Malone, R. Laubacher, and C. Dellarocas, "The collective intelligence genome," IEEE Engineering Management Review, vol. 38, no. 3, p. 38, 2010.

[22] S. Basiouka and C. Potsiou, "The volunteered geographic information in cadastre: perspectives and citizens' motivations over potential participation in mapping," GeoJournal, v. 79, no. 3, pp.343-355, 2014.

[23] P. Tobien, L. Lischke, M. Hirsch, R. Krüger, P. Lukowicz, and A. Schmidt, "Engaging people to participate in data collection," 2016, pp. 209-212.

[24] E. D. Mekler, F. Brühlmann, K. Opwis, and A. N. Tuch, "Disassembling gamification: the effects of points and meaning on user motivation and performance," 2013.

[25] J. Goncalves, S. Hosio, J. Rogstadius, E. Karapanos, and V. Kostakos, "Motivating participation and improving quality of contribution in ubiquitous crowdsourcing," $C$. Networks, vol. 90, pp. 34-48, 2015.

[26] R. I. Ogie, "Adopting incentive mechanisms for largescale participation in mobile crowdsensing: from literature review to a conceptual framework," Humancentric Computing and Information Sciences, vol. 6, no. 1, Dec. 2016.

[27] S.-W. Huang and W.-T. Fu, "Motivating crowds using social facilitation and social transparency," 2013.

[28] IDC, "Smartphone OS Market Share, 2017 Q1," 2017. [Online]. Available: https://goo.gl/zXEn6Y.

[29] M. Bergendahl, M. Magnusson, J. Björk, and M. Karlsson, "Inducing ideation collaboration through competition?" presented at the COINs15, 2015. 